\title{
Using the Lab-8 for experimentation with the human visual system
}

\author{
JOHN C. ARMINGTON \\ Northeastern University, Boston, Massachusetts 02115
}

The eyes are seldom completely at rest. Even though an attempt is made to hold them in the same position, they make frequent small movements about the point of fixation. A series of studies (Ratliff, 1965; Ditchburn, Fender, \& Mayne, 1959; Keesey, 1971) have shown that these eye movements play a crucial, although not a completely understood, role in the maintenance of vision. The importance of eye movements may be recognized by recalling that the visual system, as any sensory system, responds to a change in the stimulus. Whenever the eye moves, the retinal image sweeps across the receptors, thus producing a change in their stimulation. The change can be large enough to produce electroretinograms (ERG) (Armington, Gaarder, \& Schick, 1967) and visually evoked cortical potentials (VECP) (Gaarder et al, 1964) of sufficient amplitude for detection with average response procedures. Thus, study of these potentials in relation to eye movement can elucidate visual function.

This report will describe an apparatus for detecting responses initiated by saccadic eye movement. Basically the apparatus consists of a response-averaging computer which is triggered by eye movement. It has three component systems: (1) a system for stimulating the eye; (2) a system for recording eye movements and electrical potentials; and (3) a Lab-8 computer system for processing the response data. During experimentation, stimulation and recording necessarily proceed simultaneously. Some data analysis may be performed on-line. Complete analysis requires that the recordings be stored on tape and played back into the computer system several times. This is done at the end of the actual experimental sessions. The operation of the three component systems will now be considered separately.

\section{STIMULATOR}

The stimulator system presents the $S$ with a constant unfluctuating striped visual field. A simple Maxwellian view system of conventional design is used for this. The diagram in the upper section of Fig. 1 explains its operation. Light from a tungsten ribbon filament is imaged upon an aperture stop, AS, by a pair of lenses, L. A second pair of lenses, $L$, bring the aperture stop to a focus within the entrance pupil of the eye of the $S$. A grating is placed in the plane of the field stop and is imaged on the retina. The stimulus lamp, an 18.A $6-\mathrm{V}$ tungsten ribbon filament, is powered with a constant current source. A heat filter, $\mathrm{HF}$, is permanently mounted in the stimulus path. Stimulus luminance is controlled by interchangeable neutral density filters, F. The gratings, which are imaged upon the retina, are also interchangeable. There are no shutters or other devices in the optical path to switch the stimulus off and on or to displace it. The appearance is that of a steady striped field within a dark circular surround.

\section{RECORDING}

Simultaneous recordings are made on four channels. Electroretinograms, obtained with a contact lens electrode with a reference to the cheek, are sent through the first channel. Upon amplification, they are recorded on magnetic tape or sent directly to the data processing system. Visually evoked cortical potentials are sent through the second channel. They are picked up with a pair of standard EEG electrodes. One electrode is placed on the midline, about $1 / 2$ in. interior to the inion. The other electrode is also on the midline, but 2 in. anterior to the first. The occipital potentials are also amplified before recording on magnetic tape. Eye movements, recorded on the third channel, are obtained photoelectrically. The optical system used for this purpose is sketched in the lower section of Fig. 1. The system takes advantage of a mirror attached to the same tight-fitting contact lens that is used to record the ERG. Light, reflected from the mirror to a photocell, provides an indication of the eye movement.

The optics of the eye-movement recording system are of conventional design. Tungsten light is imaged upon the aperture stop, AS, by lenses, $L$. The aperture stop in turn is imaged on the contact lens mirror. The image of the aperture stop at the mirror is less
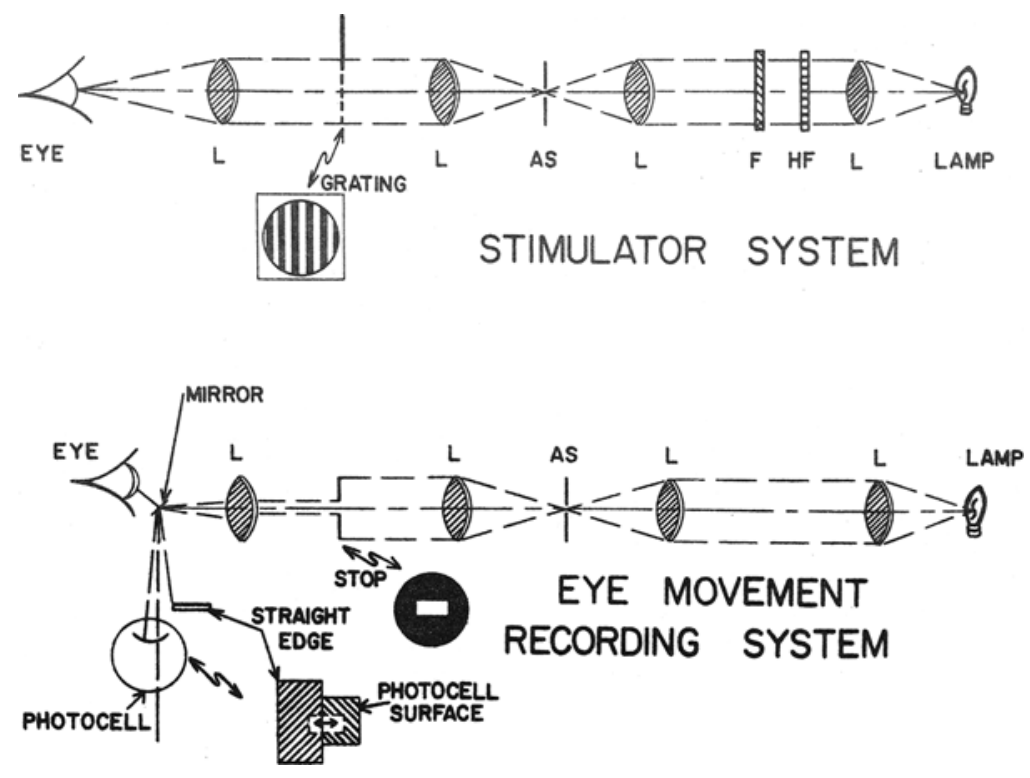

Fig. 1. Sketch of the apparatus used for stimulating the eye and for recording eye movements. 


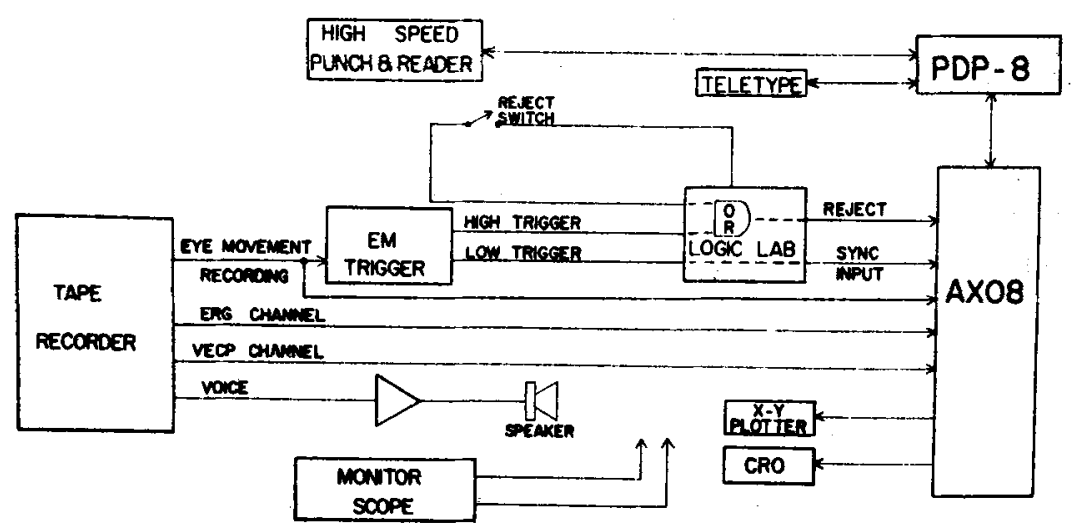

Fig. 2. Block diagram of the data-proceasing system.

than $1 \mathrm{~mm}$ in diam and is entirely contained within the mirror. A rectangular stop contained within the system is imaged on a straight edge placed directly in front of the photocell. Whenever the eye moves laterally, the image is moved so that more or less of it passes to fall on the photocell immediately behind. Thus, the photocell current provides an indication of the position of the eye. The photocell signal is amplified and recorded on the third channel of the tape recorder.

A fourth channel of the tape recorder is connected to a microphone and audio amplifier. This channel records the experimental protocol.

As it is important that the eye of the $S$ be positioned carefully in relation to the stimulator system and the eye-movement recording system, both are mounted upon the same solid base. A cross-feed mechanism, attached to this base, holds the biting board for the S. A small spot is marked in the center of the grating for fixation control. The existing system is designed to work with horizontal eye movements. The grating stripes are oriented vertically so that the largest number of receptors will receive a change in stimulation with the horizontal movement of a given size. The system could easily be modified to work with vertical eye movements as well by adding a second photocell.

\section{DATA ANALYSIS}

In the analysis of the data, a determination is made of the average ERG and the average VECP elicited by right or left saccadic eye movements of specified size. The arrangement for doing this is outlined in Fig. 2. The four tracks of the tape recorder are all operated at the same time since simultaneous averages of saccades, electroretinograms, and evoked potentials are desired. The output of the voice channel, which is just used to assist the operator in keeping track of the experimental procedure, is amplified and sent to a loudspeaker. The other three channels containing the actual data send their output to a Lab-8 computer system whose components are a PDP-8/I computer, a teletypewriter, a high-speed reader and punch, an X-Y plotter, an oscilloscope, and an AX08 laboratory peripheral unit. The last is a combination device containing a multiplexer, an analog-to-digital converter, two digital-to-analog converters, clock circuits, an array of digital input and trigger circuits which can be set by external signals to be read into the PDP-8 accumulator, and also several digital outputs. In the present situation, an external trigger circuit driven by a branch of the tape-recorder eye-movement channel produces a pulse whenever there is a saccadic eye movement above some preset amplitude. This pulse is delivered to the AX08 as a synchronous input signal. It indicates that an eye movement has occurred and that the computer should sample the three activities (namely, saccades, electroretinograms, and evoked potentials) which the tape recorder is sending to the analog-to-digital conversion system. After sampling the signals, the computer holds them until a decision is made as to whether they should be combined with the average being held within its memory. This decision depends upon the maximum amplitude achieved by the movement. If the eye movements exceed a predetermined amplitude or if the $\mathrm{E}$ signals the computer that the recording is artifactual, a rejection pulse occurs and the sampled activity is not combined with the stored average. Thus, the computer averages input activity only when saccades fall within a predetermined range of amplitudes.

A plug-in assembly of digital logic units, known as a Logic Lab, interfaces the trigger circuit with the AX08. It increases the convenience of the system. It adapts input pulses having various voltage and time characteristics to those that are compatible with the AX08. Signals appearing at its terminals are easily monitored. Plug-in construction makes it easy to adapt the Logic Lab to specific input patterns. Thus, reliability is increased and intemal control of the computer is greatly facilitated with the addition of this unit. When the eye-movement trigger circuit indicates that a saccade has occurred, a monostable pulse circuit in the Logic Lab is fired. The resulting output pulse, which serves as the synchronous input signals to the computer, exactly matches the requirements of the AX08. The trigger circuit produces a second output signal if an eye movement exceeds a second higher level. This pulse signals the computer via the AX08 to reject the sample. The reject signal for large eye movements is transmitted through an OR gate. A switch connected to a second input terminal of the OR gate may be pressed by the $\mathrm{E}$ to produce manual rejection of the average when desired.

After sampling of the recording is completed, the computer displays the average eye movement, the electroretinogram, and the visually evoked cortical potential waveforms on an $X-Y$ plotter. A cathode ray oscilloscope may also be used to display the output and to observe the response waveforms as the averaging process is under way. The high-speed reader is used to load programs into the computer, and the teletypewriter is used for control purposes. A two-beam monitor oscilloscope may be connected to any of the tape-recorder channels at various points in the trigger circuit or the Logic Lab to insure that the system is operating properly.

In practice, the reject trigger is set to respond to eye movements that are just a little larger than those which fire the lower trigger. The computer thus determines the average responses produced by eye movements lying within a narrow range of amplitudes. For complete analysis, the outcome of a single experimental session must be played into the computer several times, with the eye-movement triggers set to respond to different amplitudes. The computer must be programmed with several punched tapes during each pass. The efficiency of this operation is greatly increased with the high-speed reader, but undoubtedly could be further increased with a disk monitor system.

\section{TRIGGER}

The trigger circuit is a particularly critical part of the entire assembly. It must respond with trigger pulses whenever saccadic eye movements of 
TRIGGER CIRCUIT

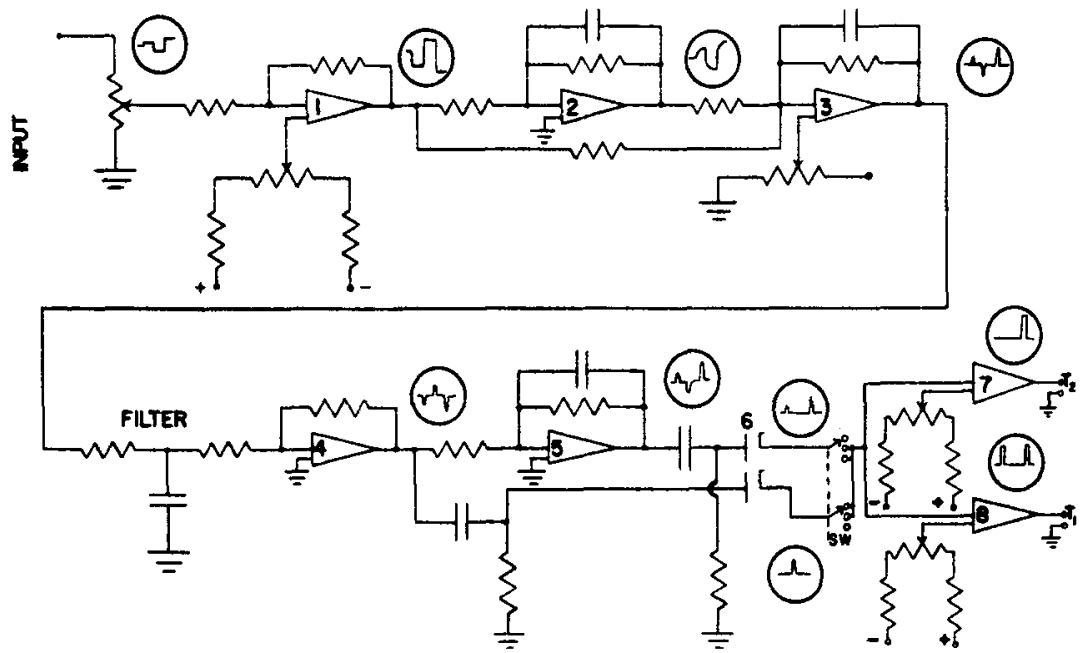

Fig. 3. Wiring diagram of the trigger circuit. The inserts show how a stepped waveform would appear at various points in a circuit.

specified amplitude appear, but it should not do so for other types of signals. Recordings of eye movements may contain many blink artifacts and other potentials which are easily confused with saccadic eye movements. Operation of the trigger circuit, which consists of a chain of operational amplifiers, is outlined in Fig. 3. The response to a series of square deflections or steps applied to the input is indicated within the circular inserts by each stage. The first stage amplifies the input. The amplified signal is differentiated and filtered by Stages 2 and 3. As a result, a rounded spike is produced at the output of Stage 3 whenever the applied signal voltage is changing rapidly. It is of opposite polarity for right and left eye movements. The spike is next amplified and applied, in part, to Stage 5, a phase inverter. The signals both at Stage 4 and Stage 5 are rectified at Stage 6 . The upper section of the rectifier tube at this stage passes pulses whenever the eye movement is in one direction and the lower half when the movements are in the opposite direction. The switch between the rectifier stage and Output

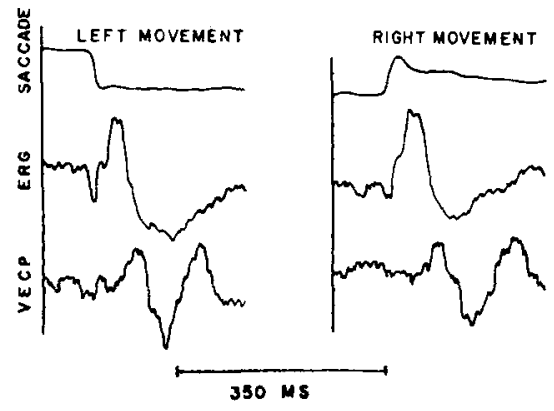

Fig. 4. Average responses recorded to left- and right-eye movements.
Amplifiers 7 and 8 thus permits selection of right, left, or right and left eye-movement spikes. Stages 7 and 8 have a very high gain but are biased below cutoff. Whenever the eye movement pulse applied to them exceeds the bias, they produce a large output potential. One of these amplifiers (T1) generates the synchronous trigger pulse; the other (T2) produces the rejection signal. The pulses produced at $\mathrm{T} 1$ and $\mathrm{T} 2$ are attenuated and transferred to the Logic Lab.

Examples of recordings produced with left and right movements are shown in Fig. 4. Samples are shown for each of the three recordings that the system produces: average eye movements, the electroretinogram, and the visually evoked cortical potential. The average eye movement has a waveform like that of single saccades observed with other recording systems (Ratliff \& Riggs, 1950). It may be noted that the right and left movements are not mirror images of one another, although both have the typical saccadic waveform. The exact pattern varies from $S$ to $S$. Electroretinograms produced by right and left eye movements across the stimulus stripes have a similar waveform and polarity, except for a small artifact appearing in the early part of the recording. This artifact is the electro-oculogram produced by the eye movement. Its polarity reverses with the direction of movement, and its amplitude increases with the extent of the movement. Visually evoked cortical potentials produced by the right and left movements are also quite similar to one another.

The averaged recordings show the activity which immediately precedes the eye movements as well as that which follows them. Synchronization pulses necessarily follow the immediate onset of eye movements with some delay in time. Rejection pulses follow with additional delay. If the program were able to average only that activity which followed a synchronization pulse and if it then had to wait to see whether a reject pulse was coming, the early portion of the response would be lost. Although, at first, it might not seem possible to average activity which comes before the synchronization pulse, this is easily achieved with the Lab-8 Advanced Averaging program used for eye-movement work by setting in a negative delay between the synchronization pulse and the start of averaging. The program samples and temporarily stores incoming data continuously. When an eye movement occurs, the program saves those samples needed for an average waveform which occurs within specified time limits before and after the synchronization signal.

The same computer system is useful for other forms of experimentation. Devices used for the study of animal behavior are readily connected to the AX08. In one experiment, the computer was used to control the stimulus and reinforcement schedules for investigating dark adaptation in squirrels (Dippner \& Armington, 1971). A Blough (1958) stimulus-reinforcement procedure was adopted, and the squirrels' dark-adaptation curves were charted on the X-Y plotter. It is clear that the system has considerable flexibility and can be applied successfully to a wide variety of visual experimentation.

\section{REFERENCES}

ARMINGTON, J. C., GAARDER, K., \& SCHICK, A. M. L. Variations of spontaneous ocular and occipital responses with stimulus patterns. Journal of the Optical Society of America, 1967. $12,1534-1539$.

BLOUGH, D. S. A method for obtaining psychophysical thresholds from the pigeon. Journal of the Experimental Analysis of Behavior, $1958,1,31-43$.

DIPPNER, R., \& ARMINGTON, J. C. A behavioral measure of dark adaptation in the American red squirrel. Psychonomic Science, $1971,24,43-45$.

DITCHBURN, R. W., FENDER, D. H., \& MAYNE, S. Vision with controlled movements of the retinal image. Joumal of Physiology, 1959, 145, 98-108.

GAARDER, K., KRAUSKOPF, J., GRAF V., KROPFL, W. J., \& ARMINGTON, J. C. Averaged brain activity following saccadic eye movements. Science, 1964 , $146,1481-1483$

KEESEY, U. T. Comparisons of human visual cortical potentials evoked by stabilized and unstabilized targets. Vision Research, 1971, 11,657-670.

RATLIFF, F. Mach bands. San Francisco: Holden-Day, 1965.

RATLIFF, F.. \& RIGGS, L. A. Involuntary motions of the eye during monocular fixation. Journal of Experimental Psychology, 1950,40, 687-701. 